\title{
Progress in the observation and modeling of turbulent multi-phase flows
}

\author{
Fabián A. Bombardelli · Hubert Chanson
}

Received: 15 February 2009 / Accepted: 16 February 2009 / Published online: 10 March 2009

(C) The Author(s) 2009. This article is published with open access at Springerlink.com

Multi-phase flows of environmental import involve the interactions of solid, gas, and/or liquid phases (Figs. 1 and 2). Figure 1 shows for instance a tidal bore, which is associated with both air entrainment in the front of the breaking bore, and with intense sediment mixing. Figure 2, in turn, shows an example of reservoir sedimentation. Despite the high loads of sediment in the river, water is diverted from the reservoir for irrigation purposes.

In the last decades, important advances have taken place in the observation and modeling of multi-phase flows. New accurate instruments to measure the air concentration in air-water flows and to analyse the motion of solid particles in water and gas; new theories to address the interactions of the diverse turbulence scales of the flow; and more powerful computers have all allowed researchers to study multi-phase flows from previously unexplored points of view. In Nature, multi-phase flows are mostly turbulent and, therefore, they are extremely complicated, with a broad range of relevant length and time scales [1]. For example, the time scales range from less than $1 \mathrm{~ms}$ for the turbulence dissipation in a small stream to about $24 \mathrm{~h}$ and $50 \mathrm{~min}$ for a tidal cycle in coastal zones (Fig. 1), and to more than 50 years for the currents controlling the balances between oxygen and carbon dioxide.

Several recent papers have remarked that the difficulties in understanding multi-phase flows stem from two basic facts [2]: (1) the phases do not distribute uniformly, and (2) the small-scale interactions may have profound effects on large-scale behaviours. These difficulties become important for the prediction of the behaviour of flows in many environmental problems and applications.

The aim of this Special Issue is to present a group of papers that: (a) summarize the state-of-the-art in the knowledge about multi-phase flows in environmental applications; (b) report recent results of research (of experimental, numerical and/or theoretical nature) on

F. A. Bombardelli ( $\square)$

Department of Civil and Environmental Engineering, University of California, 2001 Eng. III, One Shields Ave., Davis, CA 95616, USA

e-mail: fabombardelli@ucdavis.edu

H. Chanson

Division of Civil Engineering, The University of Queensland, Brisbane, QLD 4072, Australia

e-mail: h.chanson@uq.edu.au 


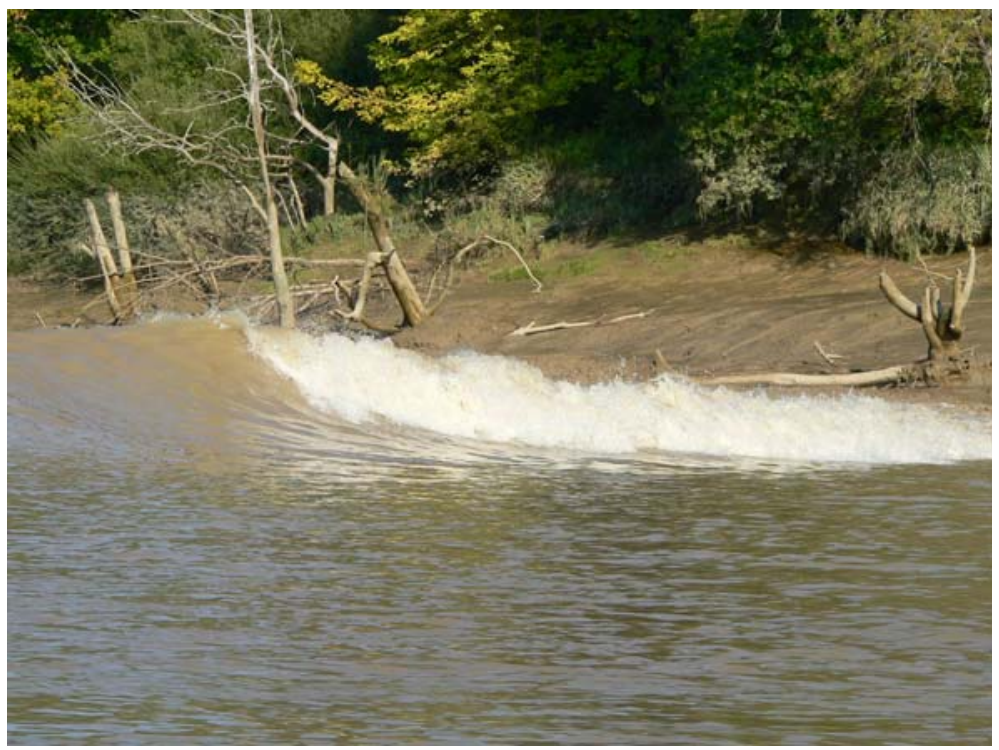

Fig. 1 Tidal bore at the Garonne River (France) on September 28, 2008, between Langoiran and Port de Podensac (bore propagation from left to right). Note the air entrainment in the breaking bore while massive sediment transport takes place next to the bank and beneath the bore front

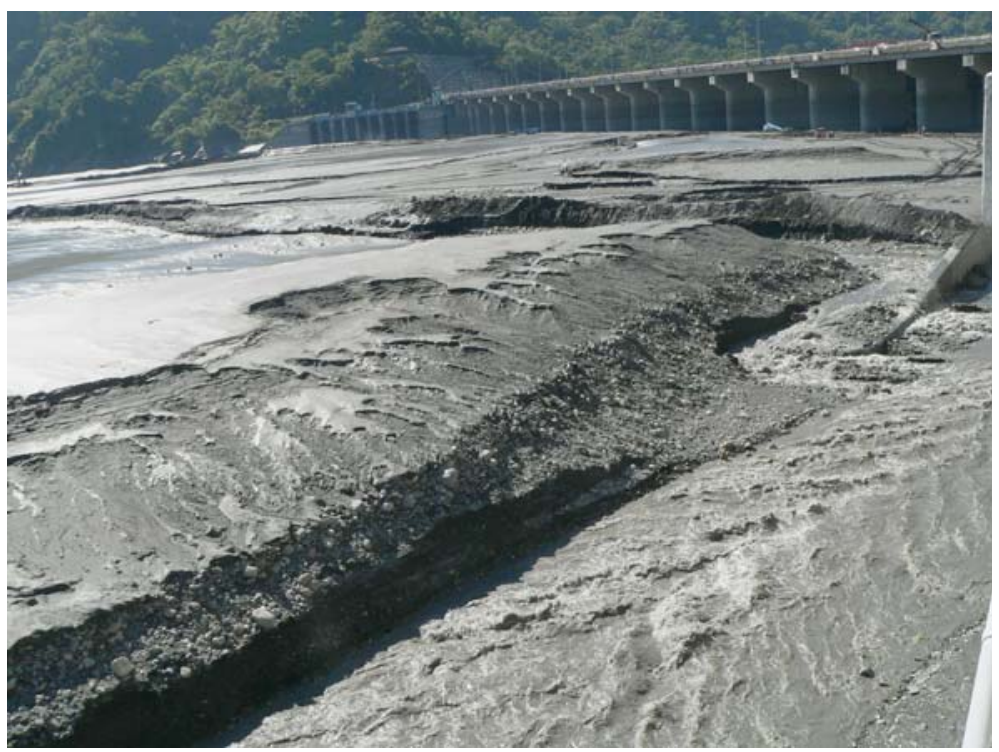

Fig. 2 Siltation upstream of the weir of the Jiji (Chi-chi) reservoir (Taiwan) on the Zhoushui River, on November 11, 2008. The reservoir sediments are regularly dredged for construction materials

environmental multi-phase flows; and (c) suggest novel pathways of analysis in the area. The current issue contains six papers accepted after a rigorous peer-review process from initially eleven papers agreed to be submitted to the journal. The deadline for paper submission was January 28, 2008, but papers were received as late as March 2008. These contributions are presented in the issue starting with the experimental papers, followed by the papers 
of theoretical and numerical nature. In both categories, there are contributions addressing gas-water as well as solid-water flows, giving an interesting balance to the topics covered herein.

The first paper discusses the effects of dynamic similarity and scale in the use of physical models of air-water flows in hydraulic structures. This topic is of paramount importance for the accurate representation of turbulence and air concentration in physical models, and for the correct extrapolation of model results to prototype conditions. The paper presents recent information of many experimental studies and discusses two mechanisms of air entrainment. The second contribution addresses the fascinating two-phase flow in hydraulic jumps, which has attracted attention for centuries. The authors present new empirical evidence regarding the bubble frequency, bubble chord length, interfacial velocity, and turbulence statistics in hydraulic jumps, for a range of Froude numbers. The third paper turns the attention to solid-water flows, focusing on the effects of particle inertia on the flow in open channels. The authors discuss results obtained with Particle Image Velocimetry (PIV) and Particle Tracking Velocimetry (PTV). The paper presents information associated with the distribution in the wall-normal direction of not only time-averaged variables but also of turbulence statistics. The paper assesses in addition the flow turbulence attenuation or enhancement produced by the presence of particles.

Turning now to the theoretical/numerical papers, the fourth contribution presents rigorous and efficient algorithms for the simulation of the motion of particles in fluid flows. The approach of the analysis is Eulerian-Lagrangian, since particles are followed individually. The fifth paper presents an Eulerian-Eulerian theoretical/numerical framework for the simulation of sediment-laden flows in open channels, including three levels of model complexity. Turbulence is treated using a well-known closure, the $k$ - $\varepsilon$ model; numerical results are compared with different datasets in terms of time-averaged flow variables as well as turbulence statistics. The last contribution assesses the influence of diverse turbulence closures on the predictions of the three models mentioned before. In both papers, the authors discuss in addition the variation of the Schmidt number, an important parameter in the prediction of sediment-laden, open-channel flows.

Lastly we would like to thank Prof. Cushman-Roisin for entertaining the idea of this Special Issue, and for allowing us to act as its Guest Editors. We also thank the numerous reviewers who significantly contributed to improve the papers presented herein, and the authors for their efforts to complete this issue in a timely manner.

Open Access This article is distributed under the terms of the Creative Commons Attribution Noncommercial License which permits any noncommercial use, distribution, and reproduction in any medium, provided the original author(s) and source are credited.

\section{References}

1. Chanson H (2004) Environmental hydraulics of open channel flows. Elsevier Butterworth-Heinemann, Oxford, UK, 483 pp

2. Hanratty TJ, Theofanous T, Delhaye J-M, Eaton J, Mclaughlin J, Prosperetti A, Sundaresan S, Tryggvason G (2003) Workshop findings. Int J Multiph Flow 29:1047-1059. doi:10.1016/S0301-9322(03)00068-5 